\title{
Is nonangiogenesis a novel pathway for cancer progression? A study using 3-dimensional tumour reconstructions
}

In recent years, the role of angiogenesis in neoplastic growth has become controversial. Initially, it was thought that the formation of new capillaries (neovascularization) usually mediated by angiogenic molecules released by tumour cells and activated macrophages was essential for all tumour growth (Perez-Atayde et al, 1997; Passalidou et al, 2002; Pezzella et al, 1997). Now there is growing evidence that in certain situations tumours can obtain sufficient blood supplies from pre-existing vascular beds to grow without angiogenesis. This form of neoplastic growth has been termed nonangiogenesis (Holash et al, 1999; Pezzella et al, 2001). A pattern of nonangiogenic growth has been described by Wesseling et al (1994) $\mathrm{Al}$ in glioblastoma multiforme and by our group in a large series of non-small cell lung carcinoma (Pezzella et al, 2001). In the latter, neoplastic cells filled the alveolar spaces (Pezzella et al, 2001) and showed no evidence of vascularization but grew by co-opting pre-existing pulmonary blood vessels. These nonangiogenic cases made up about $16 \%$ of the series (Pezzella et al, 1997) and were more aggressive clinically than the predominant angiogenic tumours (Pezzella et al, 2001).

To date these studies have given little consideration to the fact that tumour growth both angiogenic and nonangiogenic occurs in 3 dimensions. In this study, we have used computer aided threedimensional (3D) reconstructions to demonstrate the distinct differences in vascularity and morphology between the nonangiogenic and angiogenic lung tumours. We also show that in nonangiogenic tumours the integrity of the lung architecture is retained while these alveolar entrapped neoplastic cells continue to thrive without producing new vessels of their own.

*Correspondence: Dr F Pezzella;

E-mail: Francesco.pezzella@cancer.org.uk

Received 12 October 2005; revised 26 January 2006; accepted 7 February 2006

\section{MATERIALS AND METHODS}

Our model for this reconstruction is non-small cell carcinoma of the lung. Both angiogenic and nonangiogenic tissues are embedded in paraffin. The region of the tissue used for the study is $2 \mathrm{~cm}$ in depth. In all, $2005 \mu \mathrm{m}$ thick sections of paraffin-embedded tissue were cut from each case and mounted on slides coated with poly-Llysine.

\section{Clinical details of tissue samples}

Primary non-small cell lung cancer tissues (angiogenic and nonangiogenic) were obtained with informed consent from two patients who underwent radical surgical resection. The normal tissue was obtained from a patient with a lung secondary who also underwent radical resection. This research project was approved by the local ethical committee. The diagnosis was established on routine formalin-fixed paraffin-embedded material. One section was stained with haematoxylin and eosin to verify the presence of viable tumour; another was immunostained for CD34 to assess the vascular pattern as previously described.

\section{Antigen retrieval}

Antigen retrieval is as previously described by Pileri et al (1997).

\section{Immunocytochemistry}

Blood vessels and cytoskeleton on the dewaxed sections were identified by simultaneous immunostaining with $1: 500$ dilution of anti CD34 antibody QBEnd/10 (DAKO, UK) and 1:25 dilution of a pan-cytokeratin rabbit polyclonal antiserum (Novacastra, UK). The primary antibody staining was allowed to incubate for $1 \mathrm{~h}$, followed by a $5 \mathrm{~min}$ wash with TBS. Immunodetection was carried out for blood vessels and cytoskeleton by another simultaneous $1 \mathrm{~h}$ incubation with $1: 200$ dilution of both Alexa Fluor 488 goat 
anti-rabbit and Alexa Fluor 568 goat anti-mouse (Molecular Probes, USA). The immunostained sections were washed in TBS for $5 \mathrm{~min}$ and then mounted in antifade Dako Fluorescent mounting medium (DAKO, USA) containing a 1:500 dilution of 4',6-diamidine-2'-phenylindole dihydrochloride DAPI (Roche Molecular Biochemicals, UK).

\section{Image acquisition and processing}

Specific areas on immunostained sections were examined with the $\times 10$ Plan fluor objective lens of a Nikon Eclipse E600 Fluorescence microscope and photographed by an interfaced Zeiss Axiocam (Germany). Photographed images were then captured by Axiovision software in an interfaced computer. The emitting fluorescence signals were selected, respectively, by a group of filters, Dappi, FITC, Tx Red and exhibited with a resolution of 1300 by 1030 pixels of Red Green Blue (RGB). Acquired images were then batch converted from Axiovision to Photoshop where all three colours of individual images were superimposed and consecutive slide images were then stacked on top of each other for 3D reconstruction/restoration of the spatial orientation of section images. The stacked image was then imported to Imaris (Bitplane) software for the 3D rendering of the images.

\section{RESULTS}

As we expected, it was evident from the 2D picture and 3D rendering (supplement) that the architectural contour of the nonangiogenic lung tumour is a replica of the normal lung. When we compared the vascular network staining (CD34) of the normal to the nonangiogenic lung, they appeared to be indistinguishable from each other. The only difference between the normal and the nonangiogenic lung is seen when the cytokeratin staining is superimposed on CD34 staining; then the spongy like morphology of the normal lung is tumour filled in the nonangiogenic lung marked by the green cytokeratin fluorescence colour delineating these tumour cells. These nonangiogenic tumour cells are growing within the boundaries or confines of the already existing blood vessels of the lung and this pattern of the tumour growth very obviously extends beyond the surface to deep within the tissue as depicted by the 3D (supplement). Also, it is quite clear from the 3D picture (supplement) that this tumour growth pattern seen in the nonangiogenic is very distinct from the angiogenic form. The angiogenic cancer has no defined pattern of growth. Its vascular network is chaotically distributed with many blood vessels dilating twice to triple the size of adjoining vasculature. Also some of these angiogenic blood vessels appear to have blind endings while the eruption of others incite destruction of pericytes leading to the leakiness of blood vessels usually observed in angiogenic tumours.

\section{DISCUSSION}

For this experiment, we chose to use the fluorescence staining technique because its advantage over regular optical microscopy is the convenience of selectively observing the structure of the nuclei (blue), vasculature (red) and cytokeratin (green) staining individually. With this technique, there is also the option to superimpose any of the image staining upon each other. This way, we were able to trace specific regions and easily construct the 2D (Figure 1) and $3 \mathrm{D}$ morphology of the normal, nonangiogenic and angiogenic lung tumours.

What we have done with this study is to provide morphological evidence that a nonangiogenic tumour phenotype does exist. This then raises the question of whether this nonangiogenic phenotype has relevant biological differences to the angiogenic form. If this were the case, it would have major implications with respect to any antiangiogenic treatments.
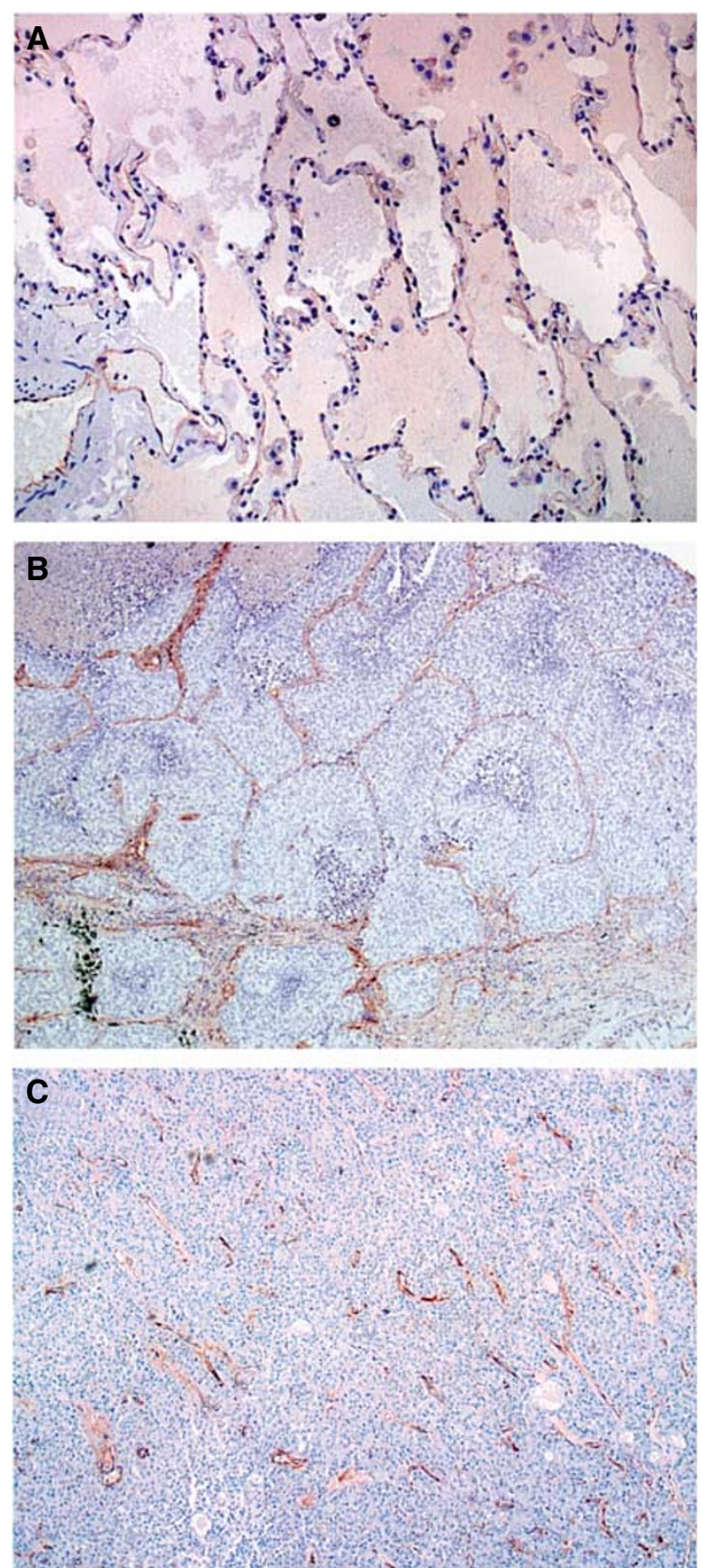

Figure I (A) H\&E slide of normal lung with a spongy appearance of the alveoli membrane, which are characteristically lined by thin blood vessels. (B) Nonangiogenic lung tumour on H\&E with the filling of alveoli by neoplastic cells, a lack of parenchymal destruction, as well as an absences of neovascularization and tumour associated stroma. The only blood vessels present are those of the alveoli septa. (C) H\&E of angiogenic lung tumour with the hallmark destruction of normal lung architecture with the production of tumour-associated stroma and of new blood vessels erratically scattered within a sea of neoplastic cells.

Studies in mice have shown that not all experimental metastases respond to antiangiogenic agents (Breast Cancer Progression Working Party, 2000). Clinical trials results suggest the possibility that some of these unresponsive tumours are of a distinct phenotype from the responsive angiogenic tumours (Gasparini et al, 2005). Studies in our laboratory have shown evidence of a variant lung tumour phenotype (the nonangiogenic form) that grows filling the alveoli without the neovascularization of angiogenesis. The idea that these alveolar filling neoplastic cells 

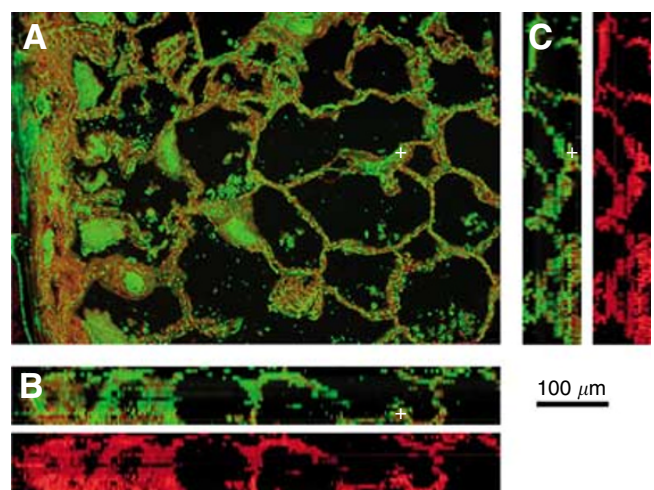

$100 \mu \mathrm{m}$

Figure 2 (A) 2D rendering of a normal lung. (B and C) Orthogonal views of the normal lung with the staining of the vascular network followed by the superimposition of cytokeratin staining.
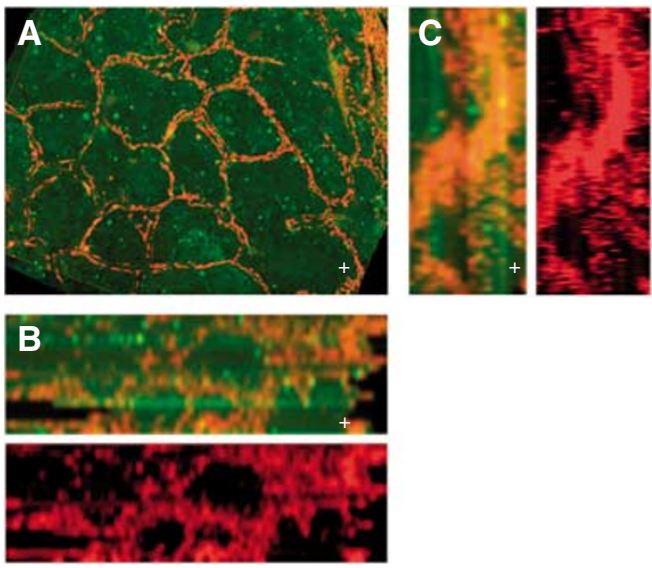

Figure 3 (A) 2D of Nonangiogenic cancerous lung with blood vessel lining still intact and similar to that of the normal lung except that in the former, the alveoli are filled with tumours. (B) 2D orthogonal view showing that the blood vessel staining of the nonangiogenic lung looks like a replica of a normal lung with no destruction to the vascular network. Followed by the superimposition of the cytokeratin staining showing neoplastic cell filled alveoli. (C) 2D orthogonal view of the nonangiogenic tumour of a large long blood vessel that remains intact and snakes through from the surface deep into the tissue with not evidence of vascular eruption.

are mostly likely thriving through co-opting of the pre-existent normal lung blood vessels is supported by our 2D (Figure 2) and $3 \mathrm{D}$ models (Figure 3) (supplemental). In contrast, the angiogenic tumours have no distinct patterns of growth and consist of heterogeneous amalgams of blind ended, tortuous blood vessels of varied sizes (Figure 4).

\section{REFERENCES}

Breast Cancer Progression Working Party (2000) Evidence for novel nonangiogenic pathway in breast-cancer metastasis. Breast Cancer Progression Working Party. Lancet 355: 1787-1788

Gasparini G, Longo R, Fanelli M, Teicher BA (2005) Combination of antiangiogenic therapy with other anticancer therapies: results, challenges, and open questions. J Clin Oncol 23: 1295-1311

Gisselsson D (2002) Tumour morphology-interplay between chromosome aberrations and founder cell differentiation. Histol Histopathol 17: $1207-1212$

Gisselsson D, Jonson T, Petersen A, Strombeck B, Dal Cin P, Hoglund M, Mitelman F, Mertens F, Mandahl N (2001) Telomere dysfunction triggers
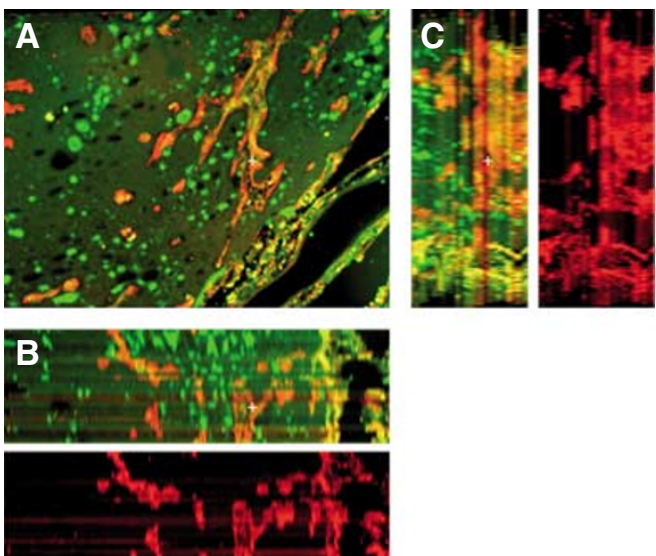

Figure 4 2D of angiogenic lung showing tortuous blood vessels of heterogeneous sizes. There evidently is no retention of the original architecture of the normal lung's blood vessel as in the nonangiogenic tumour.

Having established the existence of a putative nonangiogenic tumour phenotype, we propose that a possible explanation for this phenomena could be as a result of the evolution of tumour genes via clonal selection. Owing to hypoxic events that frequently occur during cancer cell progression, genes such as those governing efficient regulation of oxygen homeostasis could be properties clonally coselected for by evolving cancer cells (Pugh and Ratcliffe, 2003). This suggestion is supported by $\mathrm{Hu}$ et al (2005) who have described finding higher levels of genes coding for proteins involved in mitochondrial metabolism in nonangiogenic tumours. It is also known that clonal chromosomal changes found in malignant tumours have a strong correlation with tumour morphology (Gisselsson et al, 2001; Gisselsson, 2002). Hence, we suggest that by means of natural selection either through interaction between the microenvironment and the variability inherent in cell populations (Kozlov, 1996) tumours cells have evolved to this nonangiogenic form in an effort to establish a survival advantage by being able to highly regulate their mitochondria and co-opting pre-existing vessels.

\section{ACKNOWLEDGEMENTS}

We thank Michaëlla Smart for her meticulous job organising the final stage of this project. This work was supported by the Minority International Research Grant (MIRT-NIH5T37TW00067-07) and CR-UK.

Supplementary Information accompanies the paper on British Journal of Cancer website (http://www.nature.com/bjc)

extensive DNA fragmentation and evolution of complex chromosome abnormalities in human malignant tumors. Proc Natl Acad Sci USA 98: 12683 - 12688

Holash J, Maisonpierre PC, Compton D, Boland P, Alexander CR, Zagzag D, Yancopoulos GD, Wiegand SJ (1999) Vessel cooption, regression, and growth in tumors mediated by angiopoietins and VEGF. Science 284: $1994-1998$

Hu J, Bianchi F, Ferguson M, Cesario A, Margaritora S, Granone P, Goldstraw P, Tetlow M, Ratcliffe C, Nicholson AG, Harris AL, Gatter KC, Pezzella F (2005) Gene expression signature for angiogenic and nonangiogenic non-small-cell lung cancer. Oncogene 24: 1212-1219 
Kozlov AP (1996) Gene competition and the possible evolutionary role of tumours. Med Hypotheses 46: $81-84$

Passalidou E, Trivella M, Singh N, Ferguson M, Hu J, Cesario A, Granone P, Nicholson AG, Goldstraw P, Ratcliffe C, Tetlow M, Leigh I, Harris AL, Gatter KC, Pezzella F (2002) Vascular phenotype in angiogenic and nonangiogenic lung non-small cell carcinomas. Br J Cancer 86: $244-249$

Perez-Atayde AR, Sallan SE, Tedrow U, Connors S, Allred E, Folkman J (1997) Spectrum of tumor angiogenesis in the bone marrow of children with acute lymphoblastic leukemia. Am J Pathol 150: 815-821

Pezzella F, Pastorino U, Tagliabue E, Andreola S, Sozzi G, Gasparini G, Menard S, Gatter KC, Harris AL, Fox S, Buyse M, Pilotti S, Pierotti M, Rilke F (1997) Non-small-cell lung carcinoma tumor growth without morphological evidence of neo-angiogenesis. Am J Pathol 151: $1417-1423$
Pezzella F, Harris AL, Gatter KC (2001) Ways of escape: are all tumours angiogenic? Histopathology 39: $551-553$

Pileri SA, Roncador G, Ceccarelli C, Piccioli M, Briskomatis A, Sabattini E, Ascani S, Santini D, Piccaluga PP, Leone O, Damiani S, Ercolessi C, Sandri F, Pieri F, Leoncini L, Falini B (1997) Antigen retrieval techniques in immunohistochemistry: comparison of different methods. J Pathol 183: $116-123$

Pugh CW, Ratcliffe PJ (2003) Regulation of angiogenesis by hypoxia: role of the HIF system. Nat Med 9: 677-684

Wesseling P, van der Laak JA, de Leeuw H, Ruiter DJ, Burger PC (1994) Quantitative immunohistological analysis of the microvasculature in untreated human glioblastoma multiforme. Computer-assisted image analysis of whole-tumor sections. J Neurosurg 81: $902-909$ 